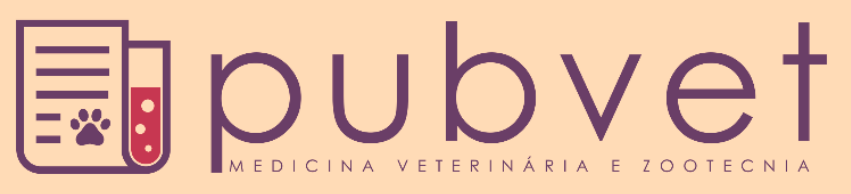

https://doi.org/10.31533/pubvet.v13n12a469.1-9

\title{
Utilização de ferramentas digitais na pecuária e extensão rural
}

\author{
Laya Kannan Silva Alves ${ }^{*} \bullet$, Gustavo Pereira Viana ${ }^{2}$, Camila Raineri $^{\circ}$ \\ 1Pós-Graduanda em Ciências na Universidade de São Paulo, Faculdade de Medicina Veterinária e Zootecnia, Departamento de Nutrição e \\ Produção Animal, Pirassununga - SP, Brasil. \\ 2Pós-Graduando em Ciências Veterinárias na Universidade Federal de Uberlândia, Faculdade de Medicina Veterinária, Uberlândia - MG, Brasil. \\ ${ }^{3}$ Professora Doutora na Universidade Federal de Uberlândia, Faculdade de Medicina Veterinária, Uberlândia - MG, Brasil. \\ *Autor para correspondência, E-mail: layakannan@usp.br
}

Resumo. Neste trabalho foi realizado um levantamento sobre os aplicativos móveis disponibilizados de forma gratuita e em língua portuguesa, voltados para a gestão no setor agropecuário e seu alcance. Para isto, foram realizadas pesquisas com palavras-chave nas lojas online Google Play e Apple Store, nas plataformas Android e iOS, respectivamente. $\mathrm{O}$ alcance foi estimado pelo número de downloads diretos para cada aplicativo móvel identificado. Foram encontrados trinta e cinco aplicativos móveis, destes, vinte e nove são pertencentes a empresas de iniciativa privada e seis oriundos de programas de esfera pública. A maioria dos aplicativos foi disponibilizada em 2017, denotando aumento da demanda no setor. Mais da metade dos aplicativos identificados são voltados para o setor de bovinocultura de corte ou leite, contudo, há indícios de uma potencial demanda para as demais categorias de aplicativos, indicada pelo alcance via downloads diretos. O comportamento observado nos dados é que aplicativos específicos tem alcance direto maior do que aplicativos genéricos de gestão. As ferramentas digitais vêm gradativamente conquistando espaço na produção animal, a internet mostra-se um excelente veículo para difundir informações técnicas geradas por pesquisadores e levá-las ao campo, atendendo à crescente demanda por informações técnicas e atuais em todos os segmentos da pecuária.

Palavras chave: agronegócio, aplicativos móveis, gestão, produtividade

\section{Use of digital tools at livestock and rural extension}

Abstract. This study verified the number of mobile applications available for free and in portuguese, aimed to management in the agricultural sector, and its reach. For this, a research whit keywords were realized on the online stores Google Play and Apple Store, of the plataforms Android and iOS, respectively. The reach was estimated by the number of direct downloads for each mobile app identified. Thirty five mobile applications was found, and twenty nine of that belong to private companies, and six of them are from public initiatives. The most of apps was uploaded in 2017, denoting the sector increased demand. Over half of the identified applications are focused in dairy and beef cattle, however, there is a evidence of a potential demand for the other categories of mobile applications, indicated by the number of direct downloads. The behaviour observed in the data was that specific applications arrived a higher reach than the generic management applications. The digital tools come gradually gaining space in the animal production, the internet is an excellent vehicle for disseminating technical information generated by researchers and bringing it to the countryside, attending the increased demand for technical and current information in all segments of livestock.

Keywords: agribusiness, mobile apps, management, productivity 


\title{
Utilización de herramientas digitales en la ganadería y extensión rural
}

\begin{abstract}
Resumen. Se realizo en este trabajo un levantamiento sobre diferentes aplicativos móviles disponibles de forma gratuita y en legua portuguesa, enfocados en la gestión del sector agropecuario y su alcance. Para ello, se realizaron búsquedas con palabras claves en tiendas en línea como Google Play y Apple Store en plataformas Android y iOS respectivamente. $\mathrm{El}$ alcance fue estimado por el número de descargas directas para cada aplicativo. Fueron encontrados 35 aplicativos móviles, de los cuales 29 son pertenecientes a empresas privadas y 6 productos de programas públicos. La mayoría de los aplicativos fueron disponibilizados en 2017, demostrando un aumento de la demanda por el sector; en donde más de la mitad de los aplicativos identificados son direccionados a el sector bovino (carne o leche). Sin embargo, existe indicios de una creciente demanda para otras especies productivas, analizado a través de las descargas directas. El comportamiento observado en los datos analizados, demostraron que los aplicativos específicos tiene mayor alcance directo, que los aplicativos de administración genéricos. Las herramientas digitales vienen ganando un importante espacio en la producción animal, convirtiéndose la internet en un excelente vehículo para difundir información técnica generada por investigadores y llevada al campo, satisfaciendo la creciente demanda de información y actualización en todos los segmentos de la ganadería.
\end{abstract}

Palabras clave: agroindustria, aplicaciones móviles, administración, productividad

\section{Introdução}

O agronegócio, além de empregar uma parcela significativa de mão de obra, contribui efetivamente para o Produto Interno Bruto (PIB) brasileiro, que apresentou em 2018 uma participação de aproximadamente $\mathrm{R} \$ 297,8$ bilhões (IBGE, 2019). Neste sentido, é essencial entender a importância e a representatividade do agronegócio perante a economia nacional, visando o desenvolvimento de novas ferramentas e metodologias que otimizem a agropecuária, a modernização da administração do setor e o aumento da produtividade (Kinouchi Filho, 2010; Undersander, 2005).

O agronegócio, assim como outros setores de impacto, apresenta dificuldades. A pecuária sofre com sistemas agroindustriais frágeis e problemas de viabilidade técnica e econômica nos setores produtivos. A eficiência produtiva média nas criações animais é baixa em várias regiões do país e um dos gargalos que contribuem com esse cenário é a dificuldade na transferência das informações geradas pela pesquisa para o campo (Ferraz \& Felício, 2010; Resende et al., 2010). O acesso às Tecnologias da Informação e Comunicação, especialmente à internet, aumentou no meio rural, favorecendo a viabilidade do uso de ferramentas digitais para desenvolvimento do Agronegócio. Assim, pode-se reconhecer a importância de explorar tais ferramentas, aumentando o alcance da difusão tecnológica e extensão rural (MCTIC, 2018).

Para que a produção animal se desenvolva no Brasil é necessário que os produtores tenham acesso à informações técnicas de forma acessível e rápida, logo, vê-se a necessidade de utilizar ferramentas como softwares e aplicativos móveis, para otimizar a produção de pequenas e grandes propriedades rurais (Resende et al., 2010). A literatura é escassa sobre o número de aplicativos e ferramentas digitais disponíveis para extensão rural, portanto este trabalho teve como objetivo realizar um levantamento dos aplicativos móveis para gestão pecuária disponíveis de forma gratuita em língua portuguesa e verificar o alcance destas ferramentas.

\section{Material e métodos}

O método aplicado para a realização deste estudo foi adaptado de Poloni \& Tomaél (2014). Os procedimentos metodológicos incluíram a revisão da literatura pertinente e o levantamento de dados primários sobre aplicativos móveis para gestão pecuária disponíveis em duas plataformas. A pesquisa buscou verificar quais aplicativos móveis estão disponíveis para auxiliar na gestão da pecuária, com acesso livre, em língua portuguesa e qual o alcance destas ferramentas.

Os dados dos aplicativos foram coletados nas lojas online Google Play e Apple Store, que são os canais oficiais de disponibilização para download de aplicativos gratuitos e pagos desenvolvidos para 
as plataformas Android e iOS, respectivamente. As buscas foram realizadas nos meses de setembro e outubro de 2018, com palavras-chave: gestão, pecuária, rebanho, bovinocultura, suinocultura, avicultura, ovinocultura, caprinocultura e agronegócio. Foram registradas também as quantidades de downloads realizados para cada aplicativo móvel identificado, dado utilizado para representar o alcance destas ferramentas.

\section{Resultados e discussão}

Buscando-se as palavras-chave gestão, pecuária, rebanho, bovinocultura, suinocultura, avicultura, ovinocultura, caprinocultura e agronegócio, foram encontrados 35 aplicativos móveis disponíveis, relacionados na Tabela 1. Em 2014, Bambini et al. (2014) realizaram um levantamento similar ao deste estudo e encontraram apenas quatro aplicativos móveis para gestão do agronegócio na plataforma Google Play. Nota-se que a maior parte dos aplicativos verificados no presente trabalho foi disponibilizada no ano 2017, o que mostra o crescimento da demanda no setor.

A difusão e apropriação das Tecnologias da Informação e Comunicação (TICs) têm sido amplamente estudadas por seu potencial transformador em termos econômicos e sociais (Affonso et al., 2015; Felippi et al., 2017; Ferreira et al., 2019; Miranda, 2015; Patel \& Sayyed, 2014; Rigi et al., 2014) O termo Tecnologias da Informação e Comunicação (TIC) refere-se à conjugação da tecnologia computacional ou informática com a tecnologia das telecomunicações e tem na Internet e mais particularmente na World Wide Web (WWW) a sua mais forte expressão (Miranda, 2007)

O levantamento TIC Domicílios, desde 2008 investiga o contingente de indivíduos com 10 anos ou mais, vivendo em áreas urbanas e rurais, que utilizaram a Internet nos três meses anteriores à realização da pesquisa. Verificou-se uma tendência de crescimento da proporção de usuários de Internet: em 2008, ela era de 39\%, ao passo que, em 2016, 61\% da população brasileira conectou-se à rede, o que equivale a 107,9 milhões de usuários no país. O estudo ressalta ainda que são observadas grandes disparidades no acesso à internet de acordo com nível de instrução, classe social, idade e áreas urbanas e rurais, mas que tais diferenças têm se atenuado entre os anos 2006 e 2016. No Brasil, 86\% da população acessou a internet todos os dias ou quase todos os dias em 2016, tendo havido um aumento significativo entre as edições de 2015 e 2016 de usuários da classe C (80\%, em 2015, contra 86\%, em 2016) e usuários das classes DE (61\%, em 2015, contra 72\%, em 2016) que se conectaram diariamente à Internet (CGI, 2017).

Dentre os 35 aplicativos encontrados durante o estudo, 29 são iniciativas de empresas privadas, representando mais de $80 \%$ dos aplicativos disponíveis de forma gratuita e em língua portuguesa, voltados para a gestão de propriedades rurais. Apenas seis aplicativos encontrados são provenientes de programas de esfera pública. A Empresa Brasileira de Pesquisa Agropecuária (EMBRAPA) é responsável pelo desenvolvimento e disponibilização de cinco destes aplicativos e apenas um foi desenvolvido por uma universidade, sendo esta, federal.

Vale ressaltar que no Brasil, a universidade gera uma grande gama de informações importantes para a população através da pesquisa, no entanto a relação entre os centros de pesquisas e universidades com a comunidade e, consequentemente, com os produtores rurais é muitas vezes ineficiente, e grande parte das informações, inovações e tecnologias geradas pelas universidades não chegam ao campo, gerando uma lacuna na transferência de conhecimento. Dessa forma, muito do conhecimento gerado não é utilizado pelos produtores rurais, o que dificulta a otimização da produção nas propriedades rurais e a comunicação entre os atores da cadeia (Valeiro \& Pinheiro, 2008).

O Ministério da Ciência, Tecnologia, Inovações e Comunicações vem expandindo o programa Internet Para Todos, em parceira com os governos municipais, estendendo a internet para localidades sem acesso à banda larga para populações em pequenas cidades do Brasil. Esta nova realidade permitirá o acesso à informação pela comunidade rural, uma fatia da população que, em grande parte, é avessa à adoção de tecnologias computacionais (MCTIC, 2018).

Conhecendo as especificidades desse público, podem ser desenvolvidos cada vez mais canais de acesso ao conhecimento gerado, às tecnologias e aos serviços, possibilitando que sejam utilizados por quem jamais teve acesso à informática, o que é facilitado com a consolidação no uso de dispositivos móveis e de mídias digitais, como aplicativos (Campanhola et al., 1998; Chaim et al., 1999; Londres, 2011; Undersander, 2005). Vários organismos internacionais têm apontado para uma associação entre o acesso às TICs e o 
desenvolvimento socioeconômico. Embora ainda existam muitas controvérsias na literatura sobre o impacto econômico da internet, por exemplo, alguns estudos empíricos explicitam uma relação positiva entre a expansão desse tipo de conexão e o crescimento econômico agregado (Galperin \& Mariscal, 2016).

Tabela 1. Aplicativos móveis em português relacionados a controle e gestão da pecuária, plataformas em que estão disponíveis para download, data de inclusão e número de downloads

\begin{tabular}{|c|c|c|c|}
\hline Aplicativo & Função & Plataforma & $\begin{array}{c}\text { Data de } \\
\text { disponibilização }\end{array}$ \\
\hline$\overline{4 \text { Milk }}$ & Gestão zootécnica de rebanho de leite & Android e iOS & $16 /$ jun/16 \\
\hline A3 Pecuária Software & Gestão de rebanho & Android & $03 / \mathrm{mar} / 16$ \\
\hline$\overline{\text { Agro Techs }}$ & Gerenciador de Agronegócio & Android & 04/dez/17 \\
\hline AGROGER 2.0 & Gerenciamento de empresas rurais & Android & $16 / j u l / 18$ \\
\hline$\overline{\text { AGROHUB }}$ & Ferramenta diária de manejo a campo & Android e iOS & Não consta \\
\hline Agrophone Suínos & Sistema móvel para suinocultura & Android & 21/out/15 \\
\hline Assistente de Avicultura & Assistente para criação e incubação de aves & Android & 27/abr/17 \\
\hline BeefTrader & Planejamento e otimização de lucro na Bovinocultura de corte & iOS & Não consta \\
\hline $\begin{array}{l}\text { Bov Control-Controle de } \\
\text { Rebanho Bovino }\end{array}$ & $\begin{array}{l}\text { Desempenho na produção de carne, leite e genética de } \\
\text { rebanho bovino }\end{array}$ & Android & 28/out/15 \\
\hline Brabov & Controle e produtividade na criação de bovinos & Android e iOS & $19 / \mathrm{fev} / 15$ \\
\hline C7 Gado de Corte - Manejo I & Sistema de registro de operações de manejo de bovinos de corte & Android & $28 /$ jan/15 \\
\hline Caderno de Campo & Manejo de pastagens & Android e iOS & 08/nov/16 \\
\hline Ceres Bovinocultura & Controle de produção leiteira de Bovinos & Android & 04/ago/17 \\
\hline CIM Custos & Cálculo de custos de sistema de produção de caprinos e ovinos & Android & Em desenvolvimento \\
\hline CIM Rebanho & Dados estatísticos e cálculos zootécnicos na produção de caprinos e ovino & Android & Em desenvolvimento \\
\hline Controle de gado & Manejo do rebanho e finanças na produção de bovinos & Android & 24/dez/17 \\
\hline Custo Fácil & $\begin{array}{l}\text { Cálculo simplificado de custo de produção para } \\
\text { integrados de sistemas de produção de aves e suínos }\end{array}$ & Android & 15/jun/16 \\
\hline Gestor+ & Gestão de rebanho Senepol & iOS & Não consta \\
\hline $\begin{array}{l}\text { Go.Farms Gestor - gestão de } \\
\text { pecuária }\end{array}$ & Controle de funcionários e serviços & Android & 29/mai/17 \\
\hline $\begin{array}{l}\text { HS Campo - Gestão de } \\
\text { rebanhos bovinos }\end{array}$ & Gestão de rebanho bovino & Android & Não consta \\
\hline JetvBov de Campo & Coleta de dados de produção de bovinos & Android e iOS & $07 / \mathrm{fev} / 17$ \\
\hline Lebov & Gerenciamento de rebanho bovino e pecuária de precisão & Android e iOS & $04 / j u l / 17$ \\
\hline Leigado Gestão de Gado Leiteiro & Gestão de propriedade produtora de bovinos de leite & Android e iOS & Não consta \\
\hline Novilho Precoce MS - ASPNP & $\begin{array}{l}\text { Acompanhamento técnico e financeiro de associados } \\
\text { produtores de carne bovina. }\end{array}$ & iOS & Não consta \\
\hline Orçamento forrageiro & Simulador de orçamento forrageiro & Android & 03/nov/17 \\
\hline Pescatec & Gestão e nutrição animal na piscicultura & Android e iOS & 26/out/17 \\
\hline Produzindo certo & Gestão ambiental de propriedade rural & Android & 17/nov/16 \\
\hline Rastrovet & Controle e gestão de rebanho & Android & 08/jul/15 \\
\hline Rebanho Mobile & Gestão de rebanho bovino & Android & 10/dez/17 \\
\hline Roda da Reprodução & Gerenciamento de rebanho na produção de bovinos de leite & Android & Não consta \\
\hline Sistema Rebanho/Gado leiteiro & Gerenciamento de gado leiteiro & Android & $23 / a b r / 14$ \\
\hline SmartGado & Controle de rebanho leiteiro & Android & $30 / a b r / 18$ \\
\hline Software Livre para Gado & Gestão de gado leiteiro e gado de engorda & Android & $15 / a b r / 14$ \\
\hline Sw Rural & Controle de rebanho bovino leiteiro & Android & $23 / a b r / 18$ \\
\hline Vet Smart & Gestão rural agrícola e pecuária & Android & 14/nov/14 \\
\hline
\end{tabular}

Neste sentido, no tocante às iniciativas em divulgação científica para o agronegócio e extensão rural, o potencial das TICs tem sido bastante explorado com a finalidade de disseminação de ferramentas de gestão e de informações ao produtor rural. Algumas das tecnologias de informação e comunicação desenvolvidas e disponibilizadas ao público são planilhas eletrônicas (Affonso et al., 2015; Raineri et 
al., 2015) softwares e aplicativos móveis (Bambini et al., 2014), blogs (Kinouchi Filho, 2010), entre outras. Bambini et al. (2014) destacam que os aparelhos celulares vêm sendo facilitadores na disseminação de informações e conhecimentos para produtores agropecuários de países em desenvolvimento, como a Índia e alguns países da África, uma vez que são equipamentos móveis, mais baratos do que computadores de mesa, mais fáceis de utilizar e de serem transportados para outros locais, conforme a conveniência, tornando-se mais acessíveis para as populações rurais, além de se tornar um importante instrumento para tomada de decisão. Desta forma, os celulares e consequentemente os aplicativos móveis, possuem um grande potencial de atuar no sentido de aumentar a qualidade de vida de populações rurais ao prover acesso à informação, serviços e produtos.

No Brasil, a Empresa Brasileira de Pesquisa Agropecuária (Embrapa) tem se destacado no contexto do desenvolvimento de softwares e aplicativos móveis para utilização na agropecuária. Como demonstrado neste levantamento sobre a pecuária, onde cinco, dos 35 aplicativos encontrados, foram desenvolvidos pela Embrapa. Para agricultura, podemos citar o aplicativo do software Gotas, desenvolvido pela Embrapa Meio Ambiente e pela Embrapa Informática Agropecuária (Chaim et al., 2006; Chaim et al., 1999; Chaim et al., 2002) com o objetivo de auxiliar os agricultores a obterem parâmetros adequados de deposição de defensivos agrícolas nos alvos desejados. O software oferece ao produtor vários parâmetros úteis para a tomada de decisão na calibração. Desde 2014 o sistema está disponível para execução em smartphones e tablets que utilizam a plataforma Android. Outro exemplo é o aplicativo Suplementa Certo (Medeiros et al., 2014a; Medeiros et al., 2014b), desenvolvido pela Embrapa Gado de Corte em parceria com a Faculdade de Computação da Universidade Federal de Mato Grosso do Sul e lançado em 2013. A finalidade do aplicativo é auxiliar o pecuarista na avaliação do benefício/custo de alternativas de suplementação do rebanho no período da seca (Medeiros et al., 2014a; Medeiros et al., 2014b).

Também o Software para Análise de Risco de Desenvolvimento de Resistência Parasitária a AntiHelmínticos em Ovinos (SARA) foi desenvolvido em 2014, sendo resultado de diversas pesquisas da Embrapa Pecuária Sudeste e da Agência Paulista de Tecnologia dos Agronegócios, além da colaboração de produtores de ovinos do Estado de São Paulo. Segundo Rosso (2014), trata-se de um software de apoio na identificação dos fatores de risco de manejo nas propriedades quanto a nematoides gastrintestinais em ovinos. Os aplicativos Gotas, Suplementa Certo e SARA não se encontram disponíveis nas plataformas Google Play e App Store, apenas para download na web.

Existe uma série de aplicativos gratuitos destinados à agricultura de precisão, a série $C 7$, que faz parte do Projeto de Desenvolvimento e Difusão de Aplicativos Android para tablets e smartphones da Universidade Federal de Santa Maria, entre eles estão os aplicativos desenvolvidos por Réquia (2013) e o C7 Nutribovinos, que consiste num sistema de cálculo de necessidades nutricionais para bovinos de corte e leite. O aplicativo o C7 Gado de Corte - Manejo I, encontrado neste levantamento faz parte do projeto e é o único aplicativo móvel encontrado neste levantamento, em língua portuguesa e gratuita, desenvolvido por uma universidade.

No presente estudo, os temas abordados pelas ferramentas levantadas são diversos, desde gestão de pessoas, como é o caso dos aplicativos Go.Farms - Gestão da Pecuária e Gestor+, a aplicativos que gerem e controlam os plantéis, sendo este de ovinos, caprinos, bovinos, suínos, aves ou até mesmo peixes, como é o caso dos aplicativos CIM Rebanho, CIM Custos, Rebanho Mobile, Brabov, Agrophone Suínos, Assistente de Avicultura, Pescatec, entre outros.

Verificou-se que mais da metade dos aplicativos de gestão pecuária encontrados nas plataformas Google Play e App Store são voltados para propriedades produtoras de bovinos, seja de corte ou leite. Entretanto, há indicativos de potenciais demandas em outros setores, verificadas pelo alcance dos aplicativos móveis levantados através do número de downloads atingido por cada categoria, como demonstra o Gráfico 1. Ao se comparar aplicativos específicos para determinado tipo de produção com aplicativos genéricos, percebe-se o maior alcance das aplicações distintivas. Tal comportamento pode ser observado por meio dos aplicativos voltados para produção de bovinos de leite e de corte, uma vez que, separadamente, atingiram maior número de downloads, quando comparados aos 4 aplicativos voltados para gestão de bovinos, sem especificar qual o produto final da produção bovina. 


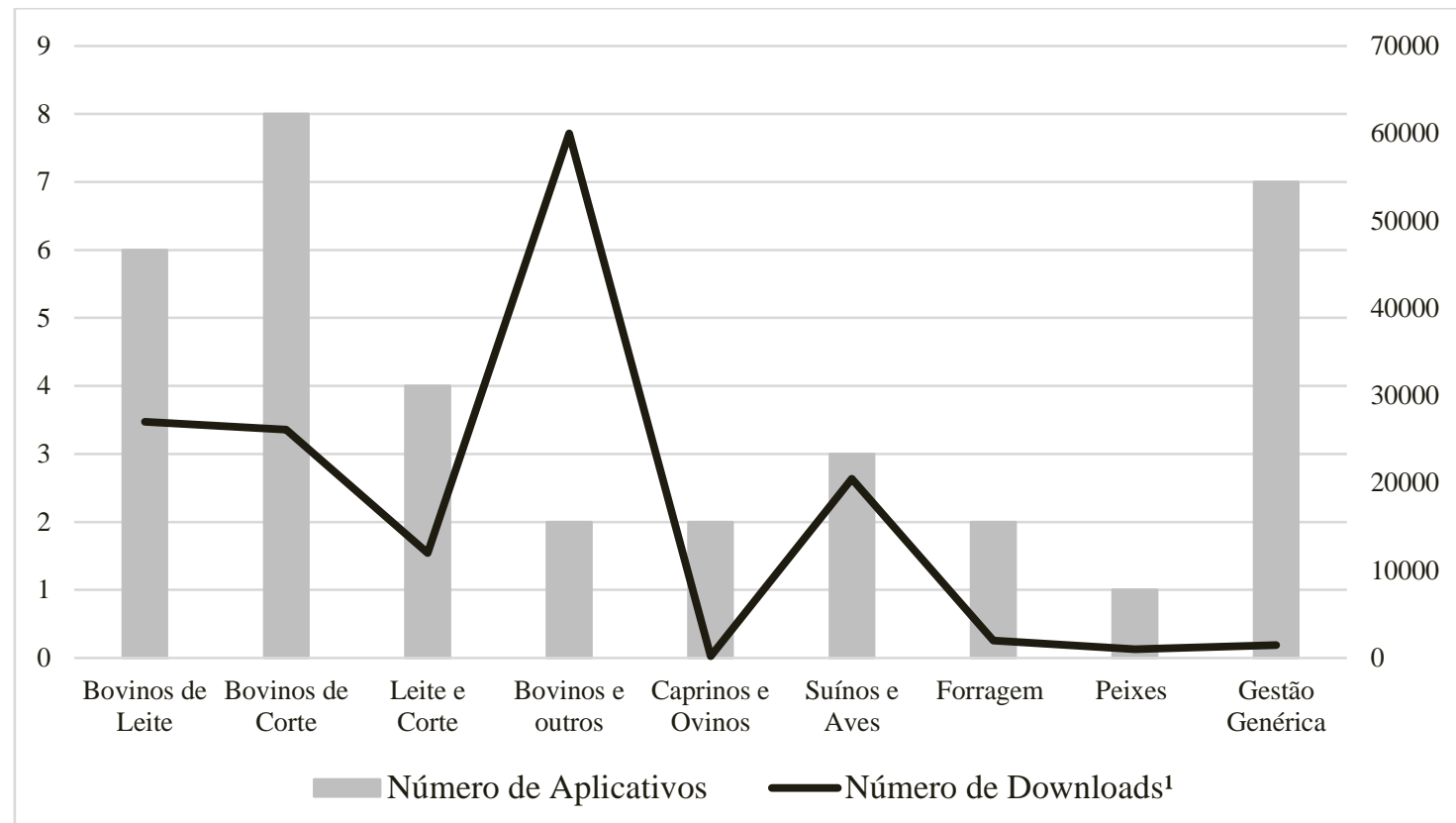

Gráfico 1. Número de aplicativos móveis encontrados por tipo de produção animal e alcance. ${ }^{1}$ Número de downloads disponíveis apenas para aplicativos disponibilizados na plataforma Google Store do sistema operacional Android. Os aplicativos disponíveis na plataforma Apple Store, do sistema operacional iOS não apresentam os dados de números de downloads, uma vez que estes se caracterizam como dados sigilosos da plataforma e não se encontram disponíveis para acesso.

A categoria "Bovinos e outros" apresenta o maior alcance, dentre os aplicativos do levantamento. São apenas dois aplicativos, sendo estes, o Software Livre para Gado, voltado para gestão de rebanhos de bovinos, ovinos, caprinos, bubalinos, camelos, lhamas e alpacas, e o aplicativo Vet Smart, que visa gestão veterinária e medicamentosa de bovinos e equinos. Esta categoria atinge a marca de 60.000 mil downloads em um período de quatro anos, o que demonstra um potencial elevado para criação de novas aplicações mobile em língua portuguesa com a finalidade de gerir rebanhos. Os aplicativos móveis voltados para produção de caprinos e ovinos apresentaram a menor abrangência ao público. O fator que explica o baixo alcance dos mesmos é o fato dos dois aplicativos disponíveis em português de forma gratuita para gestão de caprinos e ovinos, desenvolvidos pela Embrapa ainda estarem em desenvolvimento.

Algumas categorias levantadas apresentam uma demanda reprimida, como é o caso dos aplicativos voltados para gestão na avicultura e suinocultura, piscicultura e forragicultura. Foram levantados apenas três aplicativos para gestão na avicultura e suinocultura. Entretanto, essa categoria apresentou um alcance significativo de 20500 downloads. Para gestão na piscicultura e forragicultura, foram levantados apenas 2 e 1 aplicativo, com um alcance de 10000 e 2000 downloads, respectivamente. Tais dados demonstram um potencial de crescimento e oportunidade, para o desenvolvimento de ferramentas para estas categorias, uma vez que numericamente existem poucos aplicativos, porém o alcance é elevado. Já os aplicativos da categoria "Gestão genérica", variam desde gestão dos funcionários da fazenda até a gestão da propriedade como um todo. Tem-se um número elevado de aplicativos, entretanto, o alcance dessa categoria é baixo, uma vez que são sete aplicativos que juntos somam menos de 1500 downloads diretos. Neste sentido, pode-se afirmar que aplicativos mais específicos, voltados para gestão de determinada espécie ou atividade produtiva tendem a alcançar um maior número de usuários, quando comparados a aplicativos genéricos de gestão. Tais números refletem como as ferramentas digitais vêm ganhando espaço na produção animal e se mostrando um importante veículo na difusão de conhecimento técnico. Ainda assim, há um favorecimento da bovinocultura em detrimento das demais áreas da produção pecuária. Neste sentido, vê-se a necessidade da expansão no desenvolvimento de aplicativos móveis para os demais setores da pecuária, atendendo assim a demanda dos produtores rurais em todos os segmentos do agronegócio.

Apesar do foco do presente trabalho ser aplicativos em português para gestão pecuária, cabe apontar que há ferramentas disponíveis com grande variedade de aplicações, elaborados tanto por universidades 
e instituições de pesquisa quanto por empresas. Dois exemplos são citados por Bambini et al. (2014), desenvolvidos por grandes empresas do Agronegócio (Basf e New Holland). Estes aplicativos são voltados para o mercado brasileiro, e associam o fornecimento de informações meteorológicas à disponibilização de informações sobre o mercado financeiro e notícias.

Uma iniciativa no sentido de desenvolver aplicativos de utilização agropecuária foi tomada pelo projeto AWIN - Animal Welfare Indicators. Este é um projeto colaborativo que envolve pesquisadores de diversos países, inclusive brasileiros, que desenvolve pesquisa e educação na área de bem-estar animal. Com o intuito de educar proprietários, técnicos e criadores, foram criados os aplicativos WelGoat App (AWIN, 2015) e o Horse Grimace Scale Smartphone Application (HGS App) (AWIN, 2014), ambos produzidos pela equipe WP4 do projeto, sediada na Universidade Positivo, Brasil, com base nas pesquisas realizadas por outros membros do projeto, e disponibilizados gratuitamente para o sistema operacional Android no Google Play.

O HGS App foi desenvolvido pela Awin WP4 para ensinar veterinários, fazendeiros, proprietários de cavalos e cavaleiros a reconhecer e, em seguida, avaliar a dor em cavalos usando expressões faciais. Já o WelGoat App é o primeiro aplicativo especializado para investigar qualitativamente a claudicação e o crescimento excessivo de cascos em caprinos. Tanto o WelGoat App quanto o Horse Grimace Scale estão disponíveis na plataforma Google Store, do sistema operacional android.

Por conseguinte, as possibilidades de exploração de recursos digitais como aplicativos móveis para extensão rural e educação continuada são enormes. Suas vantagens incluem a facilidade de manutenção e o potencial acesso por uma infinidade de pessoas pelo mundo (Kinouchi Filho, 2010), mas a maior vantagem é que a internet provê divulgação científica para leigos e comunidades de forma descentralizada, barata e de fácil acesso (CGI, 2009; 2016).

\section{Considerações finais}

A demanda por informações técnicas e atuais nos diversos segmentos da agropecuária é crescente e a internet têm se mostrado um excelente veículo de comunicação entre os atores da cadeia. As ferramentas digitais, como os aplicativos móveis, vêm gradativamente conquistando espaço na produção animal e se mostrando eficazes na tarefa de difundir informações e conhecimento técnico gerado por pesquisadores e levá-los até o campo.

\section{Referências bibliográficas}

Affonso, E. P., Hashimoto, C. T. \& Sant'Ana, R. C. G. (2015). Uso de tecnologia da informação na agricultura familiar: Planilha para gestão de insumos. Biblios, 60.

Awin - Animal Welfare Indicators. Learning objects: The HGS (Horse Grimace Scale) App. (2014). Disponível em: http://animalwelfarehub.com/LearningMaterials/Details/91.

Awin - Animal Welfare Indicators. Learning objects: The WellGoat App. (2015). Disponível em: http://animalwelfarehub.com/LearningMaterials/Details/120.

Bambini, M. D., Luchiari-Júnior, A. \& Romani, L. A. S. (2014). Mercado de aplicativos móveis (Apps) para uso na agricultura. Paper presented at the Simpósio Nacional de Instrumentação Agropecuária, São Carlos, São Paulo, Brasil.

Campanhola, C., Rodrigues, G. S. \& Bettiol, W. (1998). Evolução, situação atual, projeção e perspectiva de sucesso de um programa de racionalização do uso de agrotóxicos no Brasil. Dialogo L, 143-45.

CGI. Comitê Gestor da Internet no Brasil. (2009). Pesquisa sobre o uso das tecnologias da informação e da comunicação no Brasil: TIC domicílios e TIC empresas 2008. São Paulo: NIC.

CGI. Comitê Gestor da Internet no Brasil. (2016). Uma introdução à governança da internet. São Paulo: CGI.

CGI. Comitê Gestor da Internet no Brasil. (2017). TIC Domicílios. São Paulo: CGI.

Chaim, A., Camargo Neto, J. \& Pessoa, M. C. P. Y. (2006). Uso do programa computacional Gotas para avaliação da deposição de pulverização aérea sob diferentes condições climáticas. Embrapa Meio Ambiente-Boletim de Pesquisa e Desenvolvimento. 
Chaim, A., Maia, A. H. N. \& Pessoa, M. C. P. Y. (1999). Estimativa da deposicao de agrotoxicos por analise de gotas. Área de Informação da Sede.

Chaim, A., Pessoa, M. C. P. Y., Camargo Neto, J. \& Hermes, L. C. (2002). Comparison of microscopic method and computational program for pesticide deposition evaluation of spraying. Pesquisa Agropecuária Brasileira, 37(4):493-496.

Felippi, Â. C. T., Deponti, C. M. \& Dornelles, M. (2017). TICs na agricultura familiar: os usos e as apropriações em Regiões do Sul do Brasil. Revista Brasileira de Gestão e Desenvolvimento Regional, 13(1):3-31.

Ferraz, J. B. S. \& Felício, P. E. (2010). Production systems - An example from Brazil. Meat Science, 84(2):238-243. doi: http://dx.doi.org/10.1016/j.meatsci.2009.06.006.

Ferreira, T. A., Carvalho, E. S., Rocha, P. M. R. \& Hofer, E. (2019). Uso e apropriação de tecnologias da informação e comunicação (TICs) como estratégia para o desenvolvimento de empreendimentos familiares no oeste do Paraná. Orbis Latina, 9(2):87-101.

Galperin, H. \& Mariscal, J. (2016). Internet y pobreza: Evidencia y nuevas líneas de investigación para América Latina: CIDE.

IBGE. (2019). PIB cresce 1,1\% em 2018 e fecha ano em $R \$$ 6,8 trilhões. Disponível em: https://agenciadenoticias.ibge.gov.br/agencia-sala-de-imprensa/2013-agencia-de-noticias/releases/23886pib-cresce-1-1-em-2018-e-fecha-ano-em-r-6-8-trilhoes. Acesso em: 09 de jul. 2019.

Kinouchi Filho, O. (2010). BLOG: Uma revista de blogs científicos com revisão por pares. Projeto de Divulgação Científica, 14.

Londres, F. (2011). Agrotóxicos no Brasil: um guia para ação em defesa da vida. Rio de Janeiro: Assessoria e Serviços a Projetos em Agricultura Alternativa.

Medeiros, B. B. L., Moura, D. J., Massari, J. M., Carvalho, T. M. R. \& Maia, A. P. A. (2014a). Uso da geoestatística na avaliação de variáveis ambientais em galpão de suínos criados em sistema "wean to finish" na fase de terminação. Engenharia Agrícola, 34(5):800-811.

Medeiros, R. M. T., Riet-Correa, F., Tabosa, I. M., Silva, Z. A., Barbosa, R. C., Marques, A. V. M. S. \& Nogueira, F. R. B. (2014b). Intoxicação por nitratos e nitritos em bovinos por ingestão de Echinochloa polystachya (capim-mandante) e Pennisetum purpureum (capim-elefante) no sertão da Paraíba. Pesquisa Veterinária Brasileira, 23(1):17-20.

MCTIC - Ministério da Ciência, Tecnologia, Inovações e Comunicações. (2018). Programa internet para todos já tem adesão de $70 \%$ dos municípios brasileiros.

Miranda, G. (2007). Limites e possibilidades das TIC na Educação. Revista da Ciência da Educação, $341-50$.

Miranda, J. C. (2015). Tecnologias da informação e desenvolvimento rural sustentável. Ciência da Informação, 44(3):381-385.

Patel, S. \& Sayyed, I. U. (2014). Impact of information technology in agriculture sector. International Journal of Food, Agriculture and Veterinary Science, 4(2):17-22.

Poloni, K. M. \& Tomaél, M. I. (2014). Coleta de dados em plataformas de redes sociais: estudo de aplicativos. Paper presented at the Atas do Workshop de Pesquisa em Ciência da Informação“Abordagens Contemporâneas na Ciência da Informação". III WPCI., Londrina, Paraná, Brasil.

Raineri, C., Stivari, T. S. S. \& Gameiro, A. H. (2015). Development of a cost calculation model and cost index for sheep production. Revista Brasileira de Zootecnia, 44(12):443-455.

Réquia, G. H. (2013). Desenvolvimento de aplicativos CR Campeiro Mobile - Caso de teste: Sistema operacional Android. Master pf Science, Universidade Federal de Santa Maria., Santa Maria, Rio Grande do Sul, Brasil.

Resende, K. T., Teixeira, I. A. M. A., Biagioli, B., Lima, L. D., Boaventura Neto, O., Junior, P. \& Deus, J. (2010). Progresso científico em pequenos ruminantes na primeira década do século XXI. Revista Brasileira de Zootecnia, 39(Sup.):369-375.

Rigi, K., Farahmand, M., Sheikhpour, S., Moradi, H. \& Keshtehgar, A. (2014). Role of information technology in agricultural development. Hournal of Novel Applied Science, 3203-205. 
Rosso, G. Software orienta produtores no controle de verminose em ovinos. 2014. Disponível em: https://www.embrapa.br/busca-de-noticias/-/noticia/1938550/software-orienta-produtores-nocontrole-de-verminose-em-ovinos. Acesso em: 13 mar. 2019.

Undersander, D. J. (2005). Role of information and information providers in technology transfer. Grassland: a Global Resource, 1351-358.

Valeiro, P. M. \& Pinheiro, L. V. R. (2008). Da comunicação científica à divulgação. Transinformação, 20(2):159-169.

Recebido: 23 de julho, 2019.

Aprovado: 6 de setembro, 2019.

Publicado: 16 de janeiro, 2020.

Licenciamento: Este artigo é publicado na modalidade Acesso Aberto sob a licença Creative Commons Atribuição 4.0 (CC-BY 4.0), a qual permite uso irrestrito, distribuição, reprodução em qualquer meio, desde que o autor e a fonte sejam devidamente creditados. 